\title{
Interdisciplinaridade no ensino de Engenharia de Software e Interação Humano-Computador com a utilização de tecnologias digitais: um relato de experiência
}

\author{
Luciana Mara Freitas Diniz ${ }^{1}$, Fischer Jônatas Ferreira ${ }^{2}$, \\ João Paulo Diniz ${ }^{3}$ \\ ${ }^{1}$ Faculdade de Pará de Minas (FAPAM) - Pará de Minas - MG - Brasil, \\ ${ }^{2}$ Universidade Federal do Ceará (UFC) - Sobral - CE - Brasil, \\ ${ }^{3}$ Universidade Federal de Minas Gerais (UFMG) - Belo Horizonte - MG - Brasil \\ luciana.diniz@fapam.edu.br, fischerferreira@ufc.br, jpaulo@dcc.ufmg.br
}

\begin{abstract}
This experience report presents the conduction of an interdisciplinary project in Software Engineering and Human-Computer Interaction in which students were responsible for documenting, modeling, and prototyping a computer application, guided by the use of digital technologies. We adopted the projectbased learning methodology as an approach in which students become active in the learning process. In addition, students answered a questionnaire about the project. Based on the student's answers, we considered the results satisfactory in terms of their declared learning, their perception of the similarity of the target project with real-world ones, the integration of complementary subjects, and the experience as a whole.
\end{abstract}

Resumo. Este relato de experiência apresenta a condução de um projeto interdisciplinar entre Engenharia de Software e Interação Humano-Computador em que os alunos foram responsáveis pela elaboração da documentação, da modelagem e da prototipação de uma aplicação computacional, guiadas pela utilização de tecnologias digitais. Foi adotada a metodologia de aprendizagem baseada em projetos como abordagem na qual os alunos se tornam ativos no processo de aprendizagem. Além disso, os alunos responderam um questionário sobre o projeto. Os resultados mostram que o aprendizado declarado, a semelhança com projetos reais, a integração de conteúdos complementares e a experiência como um todo foram consideradas satisfatórias.

\section{Introdução}

O ensino de Engenharia de Software (ES) tem alguns desafios, mas um dos principais é conciliar a teoria e a prática de conteúdos disciplinares [Souza et al. 2019, Lima et al. 2019b]. Neste sentido, preocupa-se em possibilitar aos discentes a oportunidade de experienciar a construção de software integrando conhecimentos diversos.

É sabido que as áreas de ES e Interação Humano-computador (IHC) possuem abordagens distintas sobre o desenvolvimento de sistemas, entretanto, compartilham uma interseção quanto à qualidade de uso dos mesmos [Barbosa and Silva 2010]. Sendo assim, a integração de processos, métodos e técnicas entre ambas se faz necessária e de 
grande valia para o resultado final do produto de software pois partilham aspectos complementares, como descrevem [Barbosa and Silva 2010, Lima et al. 2019a].

Na matriz curricular do curso tecnológico em Gestão da Tecnologia da Informação da Faculdade de Pará de Minas (FAPAM), a disciplina de Engenharia de Software I (ESI), com carga horária de 80 horas/aula, é ofertada aos alunos do $5^{\circ}$ período. Concomitantemente, a disciplina de IHC, com carga horária de 40 horas/aula, também é ofertada no mesmo período. A docente autora deste relato foi responsável por ministrar ambas, obrigatórias na matriz curricular do curso e, devido a este fato, surgiu a ideia de conduzir um projeto interdisciplinar, com o propósito de propiciar aos alunos uma compreensão dos conteúdos acadêmicos de forma integralizada.

A interdisciplinaridade é um dos princípios norteadores da educação profissional e tecnológica, segundo as Diretrizes Curriculares Nacionais - DCNs [CNE 2021]. Isto significa que ela deve estar inserida na prática pedagógica visando a integração dos conhecimentos, favorecendo a contextualização e a compreensão de significados nos processos de ensino e aprendizagem. Ainda, apresenta-se como componente curricular essencial no desenvolvimento de habilidades pessoais e técnicas em cursos de graduação ao contribuir para que os discentes vivenciem a prática enquanto acadêmicos [Córdova et al. 2020]. Além disso, levando-se em consideração que equipes de desenvolvimento de software, no mercado, são compostas por profissionais de múltiplas áreas, conclui-se que a interdisciplinaridade deve ser explorada em espaços acadêmicos, já que fará parte da prática profissional dos alunos futuramente [Lima et al. 2019a].

A aprendizagem baseada em projetos foi a metodologia adotada para conduzir o estudo, com o objetivo de proporcionar aos alunos uma vivência prática da teoria vista em sala de aula. Tal metodologia é classificada como ativa e tem como fundamento desenvolver a aprendizagem conceitual dos alunos por meio da prática de projetos [Mesquita et al. 2018, Lima et al. 2019b]. No ano de 2019, as disciplinas foram ministradas presencialmente mas, em 2020, as aulas ocorreram no formato remoto, adotado pela faculdade devido à pandemia do novo coronavírus. Ambas foram ofertadas no primeiro semestre de cada ano.

Sendo assim, este relato de experiência apresenta um projeto desenvolvido com alunos de graduação, no qual foram utilizadas diversas Tecnologias Digitais da Informação e Comunicação (TDIC) por meio de abordagem ativa e interdisciplinar com a integração de alguns conteúdos de ESI e IHC. Importante ressaltar que este relato se limita ao detalhamento dos aspectos do projeto relacionados à Engenharia de Software, haja visto que o relato da disciplina de IHC está descrito em publicação anterior [Diniz et al. 2020].

O documento segue com a apresentação dos métodos utilizados na Seção 2 e com detalhes do desenvolvimento e condução do projeto na Seção 3. Posteriormente, na Seção 4, são descritos os resultados e as discussões e, por fim, são apresentadas as conclusões do presente relato na Seção 5.

\section{Métodos}

A metodologia de aprendizagem baseada em projetos, em inglês Project-Based Learning - PBL, é uma iniciativa relevante que busca atender às necessidades da educação profissional pois é tida como "um novo paradigma de aprendizagem que gera e incentiva práticas 
docentes inovadoras, centradas na atuação profissional" [Sales et al. 2020], mitigando ocorrências de que muitos alunos que se formam no ensino superior não participaram de processos semelhantes aos que ocorrem no mercado de trabalho [Bessa et al. 2012].

PBL favorece a aprendizagem dos alunos quando os mesmos aplicam e desenvolvem suas ideias, haja visto que tal abordagem permite o envolvimento com problemas reais, o que trás um significado para o aprendizado [Krajcik and Blumenfeld 2005]. Isto explica o fato da motivação dos alunos ser maior quando estão inseridos em alguma prática utilizando PBL do que com os métodos tradicionais de ensino [Graaf and Kolmos 2003].

De acordo com o que descrevem [Mesquita et al. 2018], tal abordagem é caracterizada por três elementos: norteador, diferenciador e decisivo. O primeiro caracteriza-se pela resolução do problema e é possível atribuir aspectos de relevância e significado à aprendizagem, além da promoção de processos criativos e inovadores durante o desenvolvimento do projeto. A interdisciplinaridade surge como diferenciadora, por promover a integração da teoria com a prática, permite que os alunos sejam aptos a interligar diferentes áreas do conhecimentos ou áreas complementares (como é o caso de IHC e ES, no contexto deste relato). Por fim, o trabalho em equipe é o elemento decisivo que proporciona aos alunos trabalharem de forma colaborativa entre si.

No referido projeto, a convergência das disciplinas ocorre a partir da elaboração dos requisitos em ESI, os quais foram a base para a prototipação no âmbito da IHC. Durante as aulas de ambas as disciplinas, à medida em que o conteúdo era lecionado, a docente pontuava sua aplicabilidade no projeto em desenvolvimento.

Nas diretrizes curriculares publicadas pelo IEEE $^{1}$ e pela $\mathrm{ACM}^{2}$ [IEEE and ACM 2015] há recomendações para diversas disciplinas da área computacional, incluindo as de Engenharia de Software. Trechos do guia reforçam a importância da realização de trabalhos em grupo nos processos de ensino e de aprendizagem, pois auxiliam no desenvolvimento de habilidades pessoais (soft skills) tais como: comunicação, planejamento, resolução de problemas e trabalho em equipe.

Para fins de avaliação, foram concebidas as seguintes questões de pesquisa (QP):

QP1: Qual foi o aprendizado declarado pelos alunos após a realização do projeto?

QP2: Quais foram as opiniões dos alunos sobre o desenvolvimento do projeto?

QP3: Qual a relevância do projeto para os alunos?

\section{Desenvolvimento}

O projeto teve início no mês de fevereiro e término no mês de junho de 2019 e 2020, com a apresentação final do projeto pelos grupos.

De acordo com [Krajcik and Blumenfeld 2005], as ciências da aprendizagem propõem quatro ideias principais para aplicação da metodologia de aprendizagem baseada em projetos, a saber: construção criativa, aprendizagem situada, interações sociais e ferramentas cognitivas. A abordagem adotada da disciplina Engenharia de Software seguiu a aplicação desta proposta, conforme detalhado a seguir:

\footnotetext{
${ }^{1}$ Institute of Electrical and Electronic Engineers

${ }^{2}$ Association for Computing Machinery
} 
a) construção ativa: os alunos foram responsáveis pelo preenchimento do documento de especificação de requisitos (em inglês, Software Requirements Specification - SRS), disponibilizado em formato editáve ${ }^{3}$. O template utilizado foi adaptado de outros encontrados, de forma que atendesse aos critérios de simplicidade e concisão. A escolha da problemática do tema foi de livre escolha dos alunos para documentação e modelagem do sistema, proporcionando autonomia na exploração e aplicação de suas ideias, inclusive propondo soluções para problemas reais. Os próprios alunos foram os responsáveis pela elaboração dos requisitos, a partir das ideias compartilhadas em cada equipe, sem nenhuma fonte externa, mas com o auxílio da docente. O tipo de aplicação a ser projetada também ficou a cargo dos alunos, a saber: aplicação mobile, web ou desktop.

b) aprendizagem situada: para o desenvolvimento do projeto em questão, os requisitos foram especificados de maneira mais generalista. Os alunos deveriam elaborar 8 ou mais requisitos funcionais, 4 ou mais requisitos não funcionais e 4 ou mais regras de negócios, além da modelagem dos diagramas de casos de uso, da descrição dos mesmos e do diagrama de classes. Também foi solicitado a identificação de dois usuários com papeis distintos perante o sistema, além de outros stakeholders como clientes, equipe e outros sistemas integrados. Para a disciplina de IHC, os artefatos compreenderam o design de interfaces (protótipo interativo) contendo 5 telas ou mais, aplicação de 4 ou mais heurísticas de Nielsen e 3 ou mais recursos para prevenção e recuperação de erros.

c) interações sociais: os grupos foram organizados de forma que cada um tivesse de 3 a 4 integrantes. Ao todo, foram 20 participantes do projeto, sendo 9 alunos em 2019 e 11 alunos em 2020. A execução do projeto de forma individual não foi autorizada, uma vez que o trabalho em equipe é objeto da metodologia PBL. Além disso, o desenvolvimento de habilidades pessoais são importantes para a prática da ES, haja visto que o processo de desenvolvimento de software perpassa aspectos humanos e sociais [Souza et al. 2019] e a integração de profissionais de múltiplas áreas do conhecimento.

d) ferramentas cognitivas: o processo de desenvolvimento de software é apoiado por ferramentas que provêm um suporte aos profissionais na execução de suas atividades e proporcionam mais produtividade e qualidade ao produto final [Hirama 2012]. Tais ferramentas foram utilizadas no projeto para a modelagem dos diagramas da Unified Modeling Language - UML, sendo algumas delas sugeridas pela docente, quais sejam: Dia Portable, Star UML, Argo UML e Astah ${ }^{4}$, todas com licenças gratuitas para download e instalação, exceto o Astah cuja licença exige comprovação de vínculo estudantil. Um dos grupos optou pelo uso da ferramenta Draw.io ${ }^{5}$, pela praticidade na modelagem online e salvamento em repositório na nuvem. Na aulas teóricas, durante a explanação de conteúdos, foram apresentados conjuntos de slides que continham informações sobre os conceitos e a simbologia dos diagramas de casos de uso e de classes.

Nas aulas práticas presenciais, em que o intuito era a elaboração dos diagramas, a professora optou por utilizar a ferramenta Dia Portable, dado que a mesma já estava instalada nos computadores do laboratório de práticas computacionais da faculdade. Nas práticas do ensino remoto, com o recurso de compartilhamento de tela via plataforma de

\footnotetext{
${ }^{3}$ https://drive.google.com/file/d/1Wmhlo24Fv2DegjrbOkpOPQ9tL39lU-Ua/view?usp=sharing

${ }^{4}$ dia-installer.de, staruml.io, argouml-tigris-org.github.io, astah.net/downloads

${ }^{5}$ https://app.diagrams.net
} 
videochamadas Google Meet $^{6}$, a professora optou pelo uso da mesma ferramenta pela sua simplicidade nos recursos, pouco espaço de armazenamento em disco (cerca de $20 \mathrm{MB}$ ) e portabilidade para os sistemas operacionais Linux, Mac OS e Windows, favorecendo a ampla utilização pelos alunos. Além disso, tal ferramenta possui o recurso de exportar os diagramas em diversos formatos de imagem, para inclusão no documento SRS.

\subsection{Cronograma do projeto}

O planejamento das aulas expositivas e das atividades práticas dos conteúdos relacionados ao projeto teve como base o cronograma de cada disciplina, elaborado no início dos semestres. Os alunos tinham dois encontros semanais da disciplina de ESI (com duração de 1h40 cada) e um encontro da disciplina de IHC, com a mesma duração. O projeto contou com duas etapas avaliativas, com feedbacks e correções pontuais feitas nos documentos enviados pelos alunos. As entregas das etapas ocorreram conforme descrito abaixo:

$1^{\text {a }}$ etapa - entrega em meados de abril: elaboração dos requisitos funcionais, requisitos não funcionais, regras de negócio, identificação dos stakeholders, modelagem do diagrama de casos de uso e descrição dos casos de uso. Em ambas as ofertas das disciplinas, esta etapa foi valorada em 10 pontos.

$2^{\mathbf{a}}$ etapa - entrega ao final de maio: alteração do documento de acordo com as correções propostas, inclusão do diagrama de classes e prototipação das telas, conforme detalhado anteriormente. Na oferta da disciplina em 2019, esta etapa foi valorada em 10 pontos, mas em 2020 a pontuação foi de 15 pontos.

Apresentação - meados de junho: Na aula dedicada à apresentação do projeto (tanto presencial quanto remoto) os alunos criaram uma apresentação de slides (por meio de ferramenta tecnológica) contendo os artefatos produzidos. O protótipo interativo foi mostrado por cada grupo individualmente, navegando-se pelas telas por meio de links em diversos elementos. No ensino presencial foi utilizado datashow e, no ensino remoto, o recurso de compartilhamento de tela do Google Meet.

\subsection{Questionário de avaliação}

Como forma de avaliação do processo, foi enviado aos alunos um questionário online ao final dos dois semestres que compreendem este estudo, sendo o mesmo gerado na ferramenta Google Forms ${ }^{7}$, composto por duas seções. A primeira delas continha um termo de consentimento livre e esclarecido explicando o objetivo do estudo e, ao final, as opções 'Aceito participar' e 'Não aceito participar', (sendo a continuação para a próxima seção condicionada à escolha da primeira alternativa) e a segunda seção contendo 3 questões obrigatórias, sendo duas objetivas e uma discursiva.

Nas questões 1 e 2 do questionário, utilizou-se a escala Likert de 5 pontos, sendo o número 5 o maior nível e o 1 , o menor. Tal escalonamento consiste em apresentar aos respondentes afirmativas sobre determinado assunto de forma que eles optem por um nível de concordância para cada uma delas. Como resultado, cada afirmativa recebe um valor geral, com o resultado da soma das respostas individuais recebidas [Babbie 1999].

Na questão 1, os alunos deveriam assinalar a opção que mais correspondesse ao seguinte questionamento: "Como você considera o seu aprendizado sobre os conteúdos

\footnotetext{
${ }^{6} \mathrm{https}: / /$ apps.google.com/meet/

${ }^{7}$ https://drive.google.com/file/d/1iQdjquTTvkffimIjnljYRg25eVj9IxCB/view?usp=sharing
} 
disciplinares abordados no projeto interdisciplinar realizado?". Foram listados um conjunto de 10 conteúdos, sendo 7 deles relacionados à ESI e 3 à disciplina de IHC com as opções: 5 - muito satisfatório, 4 - satisfatório, 3 - normal, 2 - insatisfatório, 1 - muito insatisfatório. Já a questão 2 visava a opinião dos alunos sobre o projeto em diversos aspectos e a escala de valores teve como opções: 5 - concordo totalmente, 4 - concordo, 3 - neutro, 2 - discordo, 1 - discordo totalmente. Finalmente, a questão 3 foi a única em formato dissertativo presente no questionário, a fim de coletar as percepções dos alunos sobre o projeto como um todo, com espaço ilimitado para as respostas.

\section{Resultados e discussões}

\subsection{Projeto}

A proposta dos projetos se pautou na modelagem, documentação e protipação de uma aplicação de software, não em sua integralidade, mas tomando como base o conceito de mínimo produto viável, ou seja, com requisitos e regras de negócios básicas.

Independente da modalidade de ensino, os alunos reportaram em suas apresentações que encontraram dificuldades em ter uma visão sistêmica do software no início do projeto, que acharam complexo e um tanto quanto abstrato mapear os requisitos iniciais. À medida em que o projeto foi se desenvolvendo, com novos artefatos e incrementos, eles relataram compreender melhor o objetivo da modelagem e da documentação de um software após a prototipação do mesmo. Entretanto, os alunos que cursaram a disciplina de ES presencialmente apresentaram um documento SRS melhor escrito, organizado e formatado. Além disso, tais documentos estavam mais completos no que diz respeito ao preenchimento das descrições dos casos de uso.

Sob o ponto de vista da docente, o desempenho dos alunos na modalidade presencial (especificamente ESI) foi superior, fato que não ocorreu em relação à disciplina de IHC, cujo desempenho foi similar em ambas as modalidades, conforme relatado em [Diniz et al. 2020]. No entanto, tanto em 2019 quanto em 2020, a docente afirma que os alunos compreenderam a proposta e executaram o projeto como era esperado, em relação aos prazos e artefatos entregues.

A avaliação dos projetos foi feita exclusivamente pela docente, a partir das entregas pelos alunos e os mesmos estavam cientes que haveria uma nota única para cada equipe. Aos alunos, foi solicitado para participarem ativamente das etapas, uma vez que os conteúdos disciplinares abordados no projeto também seriam cobrados em provas, sendo eles próprios os maiores beneficiados no processo. A docente esperava que, pela prática, os alunos tivessem uma aprendizagem ativa mais significativa. A avaliação se pautou na totalidade dos artefatos entregues em cada etapa além da consistência, corretude e completude das informações. Exemplo de consistência: para 8 RFs apresentados, esperava-se que os mesmos estivessem no template na parte dos casos de uso; caso contrário, haveria perda de pontos. Se um RNF fosse adicionado incorretamente na parte das RN, também acarretaria perda de pontos. Como último exemplo, informações incompletas foram encontradas na descrição dos casos de uso, quando alguns tópicos ficaram sem preenchimento, o que também resultou em penalização na nota.

No comparativo das notas dos projetos, observa-se que no ensino presencial a média total das notas foi $18,35 \%$ superior à modalidade remota. Nas duas etapas entregues, os valores das notas refletem o que foi observado pela docente. Na modalidade 
presencial (2019), a média de nota dos trabalhos da $1^{\mathrm{a}}$ etapa foi $80 \%$ de aproveitamento e, na $2^{\mathrm{a}}$ etapa, 90\%. Os alunos fizeram a maior parte das correções propostas após a primeira entrega e acrescentaram os novos artefatos com mais completude. Na modalidade remota (2020), as correções sugeridas pela docente após a $1^{\mathrm{a}}$ entrega foram acatadas em menor número pelos alunos, principalmente em relação à descrição dos casos de uso. Na $2^{\text {a }}$ etapa, o mesmo artefato permaneceu incompleto nos projetos de 2 dos 3 grupos e os diagramas de classes continham mais inconsistências, se comparado aos entregues no ano anterior. Consequentemente, a média das notas refletiram estes problemas, com um índice de aproveitamento de $70 \%$ e $63,33 \%$ para a $1^{\mathrm{a}}$ e $2^{\mathrm{a}}$ etapas, respectivamente.

Uma dificuldade que pode ter comprometido o desempenho dos alunos teve relação com os feedbacks. No ensino presencial foi mais fácil para a docente identificar demandas, dúvidas e adicionais esclarecimentos da turma, pois há a possibilidade de perceber incompreensões, mesmo sem os discentes reportarem, seja por meio da leitura facial ou mesmo o comportamento do aluno na sala de aula. Já no ensino remoto, os feedbacks dependem da participação direta dos alunos pelo chat ou microfone, mas o feedback no ambiente remoto não tem a mesma intensidade comparado ao ensino presencial.

Ainda existe a incerteza se os alunos no ensino remoto participavam integralmente das aulas ou dividiam o horário com outras atividades paralelas. Ocorreu o fato de, por ocasião da chamada, alguns alunos não estarem presentes, embora a plataforma de videoconferência mostrasse seus perfis online (apesar do incentivo para os alunos ligarem suas câmeras, mas raramente o faziam). Possíveis distrações e atenção dividida podem interferir negativamente no rendimento e aprendizado dos alunos. No ambiente presencial, tal fato pode acontecer numa frequência menor, pois o aluno está sendo observado por colegas e pelo próprio professor.

\subsection{Questionário}

Em 2019, no ensino presencial, foram 9 os alunos que cursaram ambas as disciplinas, porém 6 responderam o questionário. Em 2020, no ensino remoto emergencial, as turmas continham 11 alunos, porém foram 8 os respondentes. Em relação aos temas dos projetos dos alunos, observou-se uma heterogeneidade e singularidade. Na apresentação dos projetos, os alunos justificaram as escolhas dos temas como segue: vivência no trabalho (temas: Consultório Médico, Gestão de Estoque e Gestão de Cartório); facilidade de compreensão do domíno (temas: Gestão de Pet Shop e Gestão de Almoxarifado) e, por fim, um problema real identificado (tema: Gestão de Posto de Saúde).

Pela experiência da docente ao longo de sete anos ministrando as disciplinas de ESI e IHC com a realização de trabalhos práticos, os alunos tendem a escolher temas que sejam parte de seus cotidianos, ou considerem o domínio fácil de compreender ou, ainda, que tenham tido experiência no trabalho. Entretanto, o tipo de aplicação escolhida pelos alunos pode ter sido influenciada pelo protótipo criado na aula destinada à manipulação da ferramenta de prototipação. No ano de 2019, a docente prototipou algumas telas de um sistema para gestão acadêmica mas, em 2020, foram prototipadas algumas telas de um aplicativo para avaliação acadêmica institucional. Todas as aplicações no ano de 2019 foram representações de sistemas desktop porém, em 2020, dois grupos optaram por desenvolver o projeto de um aplicativo mobile e somente 1 grupo optou por sistema web.

As respostas referentes às questões 1 e 2 do questionário estão representadas nas 
Tabelas 1 e 2 em números absolutos e porcentagens (por questões de arredondamento, a soma dos percentuais variam de 99 a 101). Sobre a aprendizagem declarada dos conteúdos abordados na disciplina de ESI, observou-se um resultado bastante significativo, como pode ser visto na média das respostas ao final da Tabela 1. A maioria dos alunos considera o aprendizado que tiveram sobre 'requisito funcional' e 'requisito não funcional' como positivo, sendo que as opções 'muito satisfatório' e 'satisfatório' compreendem grande parte de suas escolhas. Sobre os conteúdos 'regra de negócio' e 'stakeholders', aproximadamente $86 \%$ dos alunos consideram como muito satisfatório o nível de aprendizado que tiveram, contudo, um aluno considerou que foi insatisfatório o seu conhecimento sobre 'regras de negócio'. Em relação aos conteúdos referentes à UML (diagrama de casos de uso, descrição dos casos de uso e diagrama de classes), a maior parte dos alunos também avaliou positivamente seu aprendizado como 'muito satisfatório' ou 'satisfatório' e um aluno considerou seu conhecimento 'normal' sobre a 'descrição dos casos de uso'.

Tabela 1. Opinião sobre o aprendizado de conteúdos

\begin{tabular}{llllll}
\hline Sobre o APRENDIZADO DOS CONTEÚDOS: & $\mathbf{5}$ & $\mathbf{4}$ & $\mathbf{3}$ & $\mathbf{2}$ & $\mathbf{1}$ \\
\hline Requisito Funcional (RF) & $8(57,1 \%)$ & $4(28,6 \%)$ & $2(14,3 \%)$ & 0 & 0 \\
Requisito não funcional (RNF) & $7(50 \%)$ & $4(28,6 \%)$ & $3(21,4 \%)$ & 0 & 0 \\
Regra de negócio (RN) & $12(85,7 \%)$ & $1(7,1 \%)$ & 0 & $1(7,1 \%)$ & 0 \\
Stakeholders & $12(85,7 \%)$ & $1(7,1 \%)$ & $1(7,1 \%)$ & 0 & 0 \\
Diagrama de casos de uso (DCU) & $11(78,7 \%)$ & $3(21,3 \%)$ & 0 & 0 & 0 \\
Descrição dos casos de uso & $9(64,3 \%)$ & $4(28,6 \%)$ & $1(7,1 \%)$ & 0 & 0 \\
Diagrama de classes & $10(71,4 \%)$ & $4(28,6 \%)$ & 0 & 0 & 0 \\
Design de interfaces & $10(71,4 \%)$ & $3(21,4 \%)$ & $1(7,1 \%)$ & 0 & 0 \\
Heurísticas de Nielsen & $5(35,7 \%)$ & $4(28,4 \%)$ & $4(28,4 \%)$ & $1(7,1 \%)$ & 0 \\
Prevenção/recuperação de erros & $8(57,1 \%)$ & $3(21,4 \%)$ & $2(14,3 \%)$ & $1(7,1 \%)$ & 0 \\
\hline \multicolumn{1}{c}{ 5-muito satisfatório 4-satisfatório } & 3-normal 2-insatisfatório 1-muito insatisfatório &
\end{tabular}

Já em relação aos conteúdos disciplinares em IHC, houveram respostas mais divergentes por parte dos alunos. Especificamente sobre o conteúdo 'Heurísticas de Nielsen', este foi o único, em todos os apresentados, com o nível de expressividade menor que $50 \%$ para a opção 'muito satisfatório', de acordo com a opinião dos alunos e apenas um deles considerou 'insatisfatório' seu aprendizado. Apesar disso, grande parte dos alunos consideram a aprendizagem efetiva nos conteúdos 'prevenção/recuperação de erros' e 'design de interfaces'. Em resposta a QP1, os níveis de aprendizado declarado pelos alunos tiveram sua maioria como sendo muito satisfatório ou satisfatório, com uma média de $65,7 \%$ e $22,1 \%$ respectivamente.

A Tabela 2 mostra os resultados obtidos para a questão 2, sobre o desenvolvimento do projeto como um todo. As seis primeiras afirmativas remetem a um fator positivo. A primeira afirmativa tratou da semelhança do projeto com o trabalho/estágio dos alunos e a maioria concordou totalmente com algum nível de similaridade entre eles. A afirmação que buscou identificar se os conteúdos disciplinares foram explicados em aula teve como resultados a concordância total de 12 alunos e nenhum deles discordou. Os resultados foram um pouco diferentes sobre o projeto ter proporcionado uma visão integrada de um projeto real: a maior parte dos alunos concordou totalmente com esta asserção, porém, um aluno discordou. Ao expressarem suas opiniões sobre o resultado alcançado ter sido 
satisfatório, os alunos se dividiram entre as opções positivas (itens 4 e 5 da escala de valores). Sobre a facilidade de compreensão do template, aproximadamente $86 \%$ dos alunos concordaram com a afirmativa e $14 \%$ concordaram totalmente. Também foi uma preocupação identificar como a condução do projeto foi percebida pelos alunos e, como resultado, somente as opções 'concordo totalmente' e 'concordo', foram as escolhidas por eles, com aproximadamente $79 \%$ e $31 \%$, respectivamente.

As três últimas afirmativas apresentadas aos alunos remetiam a um fator negativo sobre o projeto como um todo. Quanto aos conteúdos do template serem confusos, 2 alunos discordaram totalmente, metade dos alunos discordou e 4 alunos manifestaram neutralidade nesta afirmativa. Sobre a afirmativa do projeto ter sido 'muito extenso para um semestre' e 'o trabalho em equipe ter sido complicado', houveram respostas para todos os valores da escala. Como resultado, a maioria dos alunos discordou de ambas (cerca de 36\%), ao passo que dois alunos concordaram totalmente com ambas (14\%).

Tabela 2. Opinião sobre o desenvolvimento do projeto

\begin{tabular}{|c|c|c|c|c|c|}
\hline Sobre o DESENVOLVIMENTO DO PROJETO: & 5 & 4 & 3 & 2 & 1 \\
\hline Identifiquei pontos semelhantes com meu trabalho/estágio & $9(64,3 \%)$ & $4(28,6 \%)$ & $1(7,1 \%)$ & 0 & 0 \\
\hline Os conteúdos disciplinares foram explicados em aula & $12(85,7 \%)$ & $2(14,3 \%)$ & 0 & 0 & 0 \\
\hline Proporcionou uma visão integrada de um projeto real & $10(71,4 \%)$ & $3(21,4 \%)$ & 0 & $1(7,2 \%)$ & 0 \\
\hline O resultado alcançado foi satisfatório & $7(50 \%)$ & $7(50 \%)$ & 0 & 0 & 0 \\
\hline O template era de fácil compreensão & $2(14,3 \%)$ & $12(85,7 \%)$ & 0 & 0 & 0 \\
\hline A sua condução contribuiu para meu aprendizado & $11(78,6 \%)$ & $3(21,4 \%)$ & 0 & 0 & 0 \\
\hline Os conteúdos do template eram confusos & $1(7,1 \%)$ & 0 & $4(28,6 \%)$ & $7(50 \%)$ & $2(14,3 \%)$ \\
\hline Muito extenso para um semestre & $2(14,3 \%)$ & $1(7,1 \%)$ & $3(21,4 \%)$ & $5(35,7 \%)$ & $3(21,4 \%)$ \\
\hline Trabalhar em equipe foi complicado & $2(14,3 \%)$ & $3(21,4 \%)$ & $3(21,4 \%)$ & $5(35,7 \%)$ & $1(7,1 \%)$ \\
\hline
\end{tabular}

Para responder à QP2, sobre as opiniões dos alunos em relação ao desenvolvimento do projeto, fica claro que prevaleceu a concordância sobre aspectos positivos no que diz respeito à condução, explicação e integração de conteúdos, além do template utilizado e a analogia com o mercado de trabalho.

Já na questão 3 do questionário, os alunos tinham um espaço ilimitado para expressarem suas opiniões a respeito do projeto, resultando em diferentes tipos de respostas. Os conteúdos de todas elas foram analisados visando identificar características semelhantes entre as opiniões dos alunos, para posterior classificação e agregação, conforme propõe [Bardin 2016]. Assim, com base na análise de conteúdo, foram criadas 5 categorias para representar ocorrências similares, como pode ser visto na Figura 1.

Nas respostas dos alunos, uma mesma frase poderia haver representatividade para mais de uma categoria, como na resposta do Aluno A: "carrego comigo o aprendizado e posso ver claramente no dia a dia em meu trabalho". Após a análise, tal resposta somou uma ocorrência tanto para a categoria "aprendizado"quanto para a de similaridade com o "mercado de trabalho". Já o Aluno B escreveu: "Trabalhar em equipe foi ótimo e simulou bem uma situação de trabalho real". Para tal, foram incluídas ocorrências nas categorias de "trabalho em equipe"e analogia com o "mercado de trabalho". Em resposta à QP3, grande parte dos alunos percebeu que o desenvolvimento do projeto contribuiu para o aprendizado deles e, mais ainda, que foi semelhante às suas práticas profissionais.

Acredita-se que esta abordagem foi bem sucedida pelo baixo número de alunos 


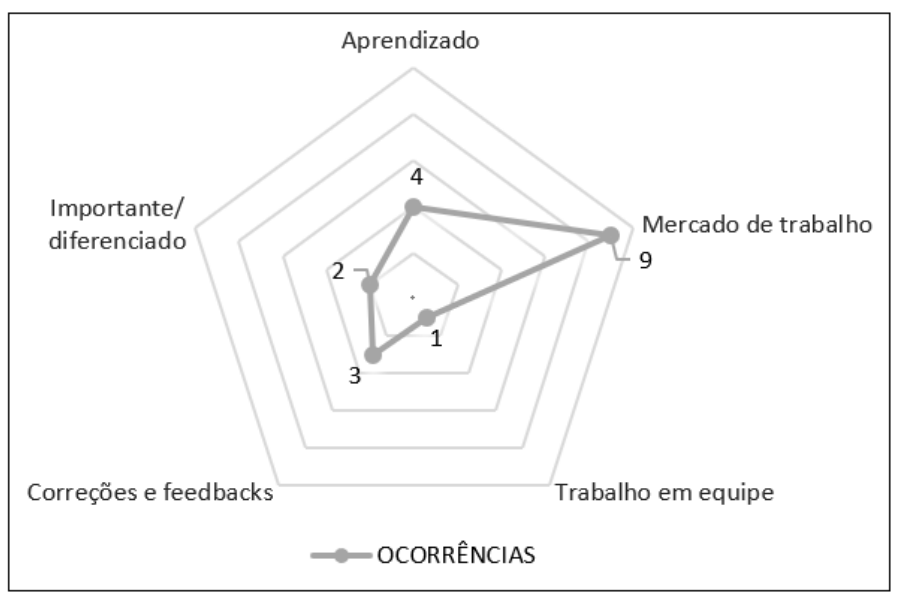

Figura 1. Categorização das respostas dos alunos.

nas turmas, a oferta das disciplinas de ESI e IHC terem sido concomitantes e, ainda, a docente ser a responsável por ministrar ambas. Em outros contextos, vários empecilhos relacionados aos itens descritos poderiam ter influenciado negativamente o desenvolvimento de um projeto com esta configuração.

\section{Conclusão}

Com o intuito de proporcionar aos alunos uma atividade prática que simulasse projetos reais no mercado de trabalho e favorecer a compreensão de conteúdos complementares com uma configuração mais abrangente, foi proposta a realização do projeto interdisciplinar e adoção da metodologia PBL com a utilização das TDIC.

Os resultados foram considerados satisfatórios quanto à aprendizagem declarada dos alunos em relação aos conteúdos disciplinares trabalhados no projeto. O template usado foi considerado de fácil compreensão, o que possibilita a sua reutilização nos próximos semestres e até mesmo em outros estudos. E, finalmente, os alunos concordaram que o desenvolvimento do projeto interdisciplinar contribuiu para o aprendizado, proporcionou visão integrada de um projeto real e, ainda, permitiu a identificação de semalhanças e vivência em trabalhos ou estágios.

Uma possível ameaça identificada é que alguns alunos já trabalhavam ou estagiavam na área de suporte ou desenvolvimento, o que pode ter influenciado no aprendizado, nas notas e no projeto bem sucedido. Além disso, devido à carga horária de ambas as disciplinas e para não comprometer a conclusão do projeto, optou-se por não incluir a implementação dos sistemas como um artefato entregável. Para mitigar os efeitos do tempo, foi definido que os projetos se limitariam à entrega dos protótipos funcionais.

Como trabalhos futuros, sugere-se que haja um comparativo entre as turmas em duas ofertas distintas: em uma delas utilize a metodologia PBL e em outra não, mantendo a interdisciplinaridade. Haja visto que o número de alunos das turmas geralmente é reduzido, talvez dividir a turma não seja muito interessante para a condução do projeto.

Espera-se que este tipo de abordagem prática e interdisciplinar seja mais aplicada e difundida entre as disciplinas complementares de cursos de tecnologia, a fim de possibilitar aos discentes uma visão integrada por meio da simulação de projetos reais. 


\section{Referências}

Babbie, E. (1999). Métodos de pesquisas de survey, volume 1. Ed. da UFMG Belo Horizonte.

Barbosa, S. and Silva, B. (2010). Interação Humano-Computador. Elsevier Brasil.

Bardin, L. (2016). Análise de Conteúdo. Edições 70.

Bessa, B., Cunha, M., and Furtado, F. (2012). Engsoft: Ferramenta para simulação de ambientes reais para auxiliar o aprendizado baseado em problemas (pbl) no ensino de engenharia de software. In Anais do XX Workshop sobre Educação em Informática. Curitiba-PR.

CNE (2021). Diretrizes curriculares nacionais gerais para a educação profissional e tecnológica. Conselho Nacional de Educação. Resolução CNE/CP No 1, de 5 de janeiro de 2021.

Córdova, P. R., Baade, J. H., and dos SANTOS, A. M. (2020). Problem-based learning (pbl) e interdisciplinaridade no ensino-aprendizagem de engenharia de software. $R e$ vista Ibero-Americana de Estudos em Educação, pages 2078-2093.

Diniz, L. M. F., Pereira, M. V., de Paula, L. J. D., and de Melo Silva, E. C. (2020). Aprendizado baseado em projetos em ihc (presencial e remoto): prototipação segundo as heurísticas de nielsen. In Anais Estendidos do XIX Simpósio Brasileiro de Fatores Humanos em Sistemas Computacionais, pages 13-18. SBC.

Graaf, E. D. and Kolmos, A. (2003). Characteristics of problem-based learning. International Journal of Engineering Education, 19(5):657-662.

Hirama, K. (2012). Engenharia de software: qualidade e produtividade com tecnologia. Elsevier Brasil.

IEEE and ACM (2015). Software engineering 2014: Curriculum guidelines for undergraduate degree programs in software engineering. Disponível em: https://www.acm.org/binaries/content/assets/education/ se2014 .pdf. Acesso em: 24 Jul. 2021.

Krajcik, J. S. and Blumenfeld, P. C. (2005). Project-Based Learning, page 317-334. Cambridge Handbooks in Psychology. Cambridge University Press.

Lima, I. F. G. d., Melo, M. C. d., da Silva, W. E., and Falcão, T. P. (2019a). Interdisciplinaridade e ihc: Possibilidades no contexto da licenciatura em computação. In Anais Estendidos do XVIII Simpósio Brasileiro sobre Fatores Humanos em Sistemas Computacionais, pages 82-87. SBC, SBC OpenLib.

Lima, J. V., Júnior, M. d. M. A., Moya, A., Almeida, R., Anjos, P., Lencastre, M., Fagundes, R. A. d. A. F., and Alencar, F. (2019b). As metodologias ativas e o ensino em engenharia de software: uma revisão sistemática da literatura. In Anais do Workshop de Informática na Escola, volume 25, pages 1014-1023.

Mesquita, D., Chagas, R., Lima, R., and Chagas, J. (2018). Assessment models in two project based learning (pbl) approaches: an exploratory study. 
Sales, A. B. d., Serrano, M., and Serrano, M. (2020). Aprendizagem baseada em projetos na disciplina de interação humano-computador. Revista Ibérica de Sistemas e Tecnologias de Informação, (37):49-64.

Souza, M., Moreira, R., and Figueiredo, E. (2019). Students perception on the use of project-based learning in software engineering education. In Proceedings of the XXXIII Brazilian Symposium on Software Engineering, pages 537-546. ACM. 